The Influence of the Asymptotic Regime on the RS-IMEX

Peer-reviewed author version

KAISER, Klaus \& SCHUETZ, Jochen (2017) The Influence of the Asymptotic

Regime on the RS-IMEX. In: Quintela, Peregrina; Barral, Patricia; Gómez, Dolores;

Pena, Francisco J.; Rodríguez, Jerónimo; Salgado, Pilar (Ed.). ECMI 2016: Progress

in Industrial Mathematics at ECMI 2016, Springer,p. 55-66.

DOI: 10.1007/978-3-319-63082-3_7

Handle: http://hdl.handle.net/1942/25935 


\title{
The Influence of the Asymptotic Regime on the RS-IMEX
}

\author{
Klaus Kaiser and Jochen Schütz
}

\begin{abstract}
In this work, we investigate the performance and explore the limits of a novel implicit-explicit splitting [6] for the efficient treatment of singularly perturbed ODEs. We consider a singularly perturbed ODE where, based on the choice of initial conditions, the unperturbed equation does not necessarily describe the behavior of the perturbed one accurately. For the splitting presented in [6], this has a tremendous influence as it explicitly depends on the solution to the unperturbed equation. That this indeed poses a problem is shown numerically; but also the remedy of using the 'correct' asymptotics is presented. Comparisons with a fully implicit and a standard implicit-explicit splitting are shown.
\end{abstract}

\section{Introduction}

Computing the solution to a singularly perturbed differential equation accurately and efficiently is a task that is ubiquitous in many scientific applications. One class of methods that is able to deal with such equations are IMEX schemes [1], 2]. At the very core of these schemes is a suitable identification of stiff terms (that should be treated in an IMplicit manner) and non-stiff terms (that should be treated in an EXplicit manner). Such a splitting of the equation can have tremendous influence on the accuracy and the efficiency of an IMEX scheme. Over the past few years, a splitting based on the unperturbed solution (the so-called reference solution, hence $R S$-IMEX splitting) has been developed, and it has been tested, amongst others, on an ODE and a PDE example [ [7, 8]. In all the applications, the solution to the

\footnotetext{
Klaus Kaiser

IGPM, RWTH Aachen University, Templergraben 55, 52062 Aachen, Germany, e-mail: kaiser(d) iqpm.rwth-aachen.de

Jochen Schütz

Faculty of Sciences, University of Hasselt, Agoralaan Gebouw D, 3590 Diepenbeek, Belgium, e-mail: jochen.schuetzduhasselt.be
} 
perturbed problem was a very close approximation to the unperturbed solution. This is not necessarily always the case for finite perturbation parameter, even if there is convergence for this parameter to zero. In the present work, we therefore try to give a glimpse on the behavior of the RS-IMEX splitting for a singularly perturbed system that has a richer structure. As a prototype equation, we consider the one suggested and analyzed in [5], namely

$$
\frac{\mathrm{d}}{\mathrm{d} t}\left(\begin{array}{c}
v \\
z \\
w
\end{array}\right)=\left(\begin{array}{c}
\frac{1}{\varepsilon}\left(-z+f_{2} v^{2}+f_{3} v^{3}\right) \\
v-w \\
\sqrt{\varepsilon}\left(\mu-g_{1} z\right)
\end{array}\right),
$$

with, in analogy to [5], $f_{2}=\frac{3}{2}, f_{3}=-1, g_{1}=\frac{1}{2}$ and $\mu=\frac{4}{100}$. It is fairly intuitive to see that the $\varepsilon \rightarrow 0$ limit (if there is any) fulfills the differential-algebraic equation

$$
\frac{\mathrm{d}}{\mathrm{d} t}\left(\begin{array}{c}
0 \\
z_{(0)} \\
w_{(0)}
\end{array}\right)=\left(\begin{array}{c}
-z_{(0)}+f_{2} v_{(0)}^{2}+f_{3} v_{(0)}^{3} \\
v_{(0)}-w_{(0)} \\
0
\end{array}\right)
$$

For the initial conditions we consider in this work, which are always of wellprepared form

$$
z(t=0)=f_{2} v(t=0)^{2}+f_{3} v(t=0)^{3},
$$

the solution to this last equation has a fairly simple structure, in particular, it exists over the whole real axis with a constant limit state as $t \rightarrow \infty$. The solution for $\varepsilon>$ 0 , on the other hand, has a quite rich structure (see e.g. [5] for a more thorough discussion of the equation). According to [5], we choose $w(t=0)=-\frac{1}{100}$ and the values of $v(t=0)$ are chosen as \pm 0.1 . All computations are done up to $t_{\text {end }}=10$.

In Fig. W the solutions for different values of $\varepsilon$ are plotted. We can observe that in all cases, for 'large' values of $\varepsilon$, a peak in the solution is present which disappears for $\varepsilon \rightarrow 0$. For the case $v(t=0)=0.1$ we observe that the reference solution which is computed by (DI), is not the limit we observe in the solution. Even more, we observe that there is an initial boundary layer (see Fig. $\square$ for a zoom of Fig. 四) for the case $v(t=0)=0.1$. The conclusion that the solution converges towards the reference solution for $v(t=0)=-0.1$ springs to mind.

Fig. 1 Left: $v(t=0)=-0.1$, right: $v(t=0)=0.1$

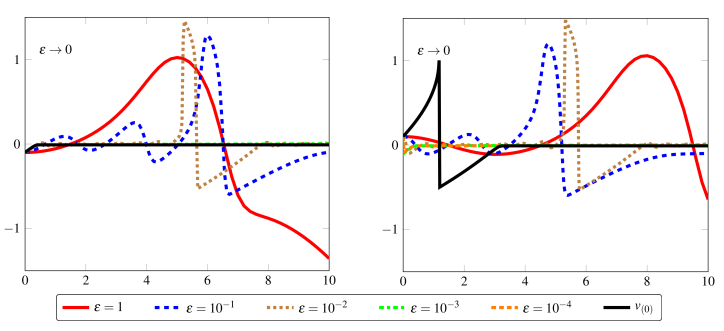


Fig. 2 Zoom into Fig. 田. Left: $v(t=0)=-0.1$, right: $v(t=0)=0.1$. Note the initial layer for the case on the right.

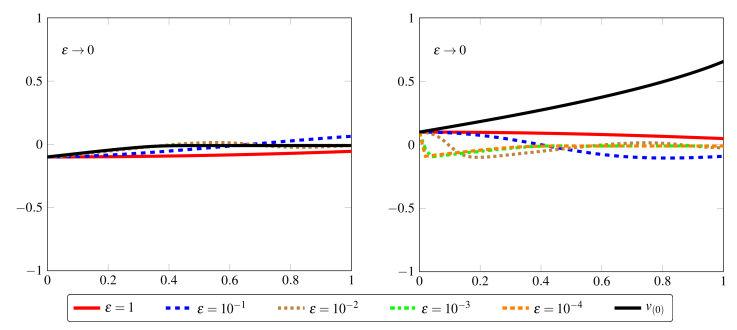

In what follows, we take different IMEX splittings and compare their behavior. Naturally, our focus is on the behavior of the RS-IMEX splitting and its performance in comparison to the other schemes. In particular, how does it perform if there is no valid asymptotic solution or if the solution converges towards a different reference solution?

\section{Comparison of Splittings}

In this section we consider three different splittings, see Table प1:

- A 'canonical' splitting (SPLIT), where the $\mathscr{O}\left(\frac{1}{\varepsilon}\right)$-terms are treated implicitly. A similar splitting was used in [3] for van der Pol's equation.

- The RS-IMEX splitting, where the reference solution is approximately computed by a fully implicit method, see [6] for details.

- A fully implicit method.

Numerical computations are run for all these splittings with IMEX Euler [1]] and IMEX BPR-353 [4] method.

Table 1 Overview of different splittings used. Left: implicit, right: explicit part.

\begin{tabular}{lll}
\hline Splitting & Implicit part & Explicit part \\
\hline SPLIT & $\left(\begin{array}{c}\frac{-z+f_{2} y^{2}+f_{3} y^{3}}{\varepsilon} \\
0 \\
0\end{array}\right)$ & $\left(\begin{array}{c}0 \\
y-w \\
\sqrt{\varepsilon}\left(\mu-g_{1} z\right)\end{array}\right)$ \\
Implicit & $\left(\begin{array}{c}\frac{-z+f_{2} y^{2}+f_{3} y^{3}}{\varepsilon} \\
y-w \\
\sqrt{\varepsilon}\left(\mu-g_{1} z\right)\end{array}\right)$ & $\left(\begin{array}{l}0 \\
0 \\
0\end{array}\right)$ \\
RS-IMEX & $\left(\begin{array}{c}-z+f_{2} 2 y_{(0)} y+3 f_{3} y_{(0)}^{2} y-f_{2} y_{(0)}^{2}-2 f_{3} y_{(0)}^{3} \\
\varepsilon-w \\
y-1 \\
\sqrt{\varepsilon}\left(\mu-g_{1} z\right)\end{array}\right)$ & $\left(\begin{array}{c}\frac{f_{2} y^{2}+f_{3} y^{3}-f_{2} 2 y_{(0)} y-3 f_{3} y_{(0)}^{2} y+f_{2} y_{(0)}^{2}+2 f_{3} y_{(0)}^{3}}{\varepsilon} \\
0 \\
0\end{array}\right.$ \\
\hline
\end{tabular}


Decent Asymptotics: $\mathbf{v}(\mathbf{t}=\mathbf{0})=-\mathbf{0 . 1}$

First, the easier case is considered, see also Fig. W. In particular, there is an $\varepsilon \rightarrow 0$ asymptotic. Figs. [3] and 0 show convergence results for IMEX Euler and IMEX BPR-353, respectively. All methods yield the desired convergence order, but the peak $\left(\varepsilon \geq 10^{-2}\right)$ makes it somewhat difficult. Furthermore, both SPLIT and RSIMEX have convergence problems for large values of $\Delta t$. For $\Delta t \rightarrow 0$, the RS-IMEX splitting produces approximations with a smaller error, and the error curve is generally smoother. Last but not least, there are stability problems for SPLIT, see Fig. [] and the RS-IMEX splitting, see Fig. W, for large values of $\varepsilon$. This behavior has also been observed for the RS-IMEX method presented in [6].

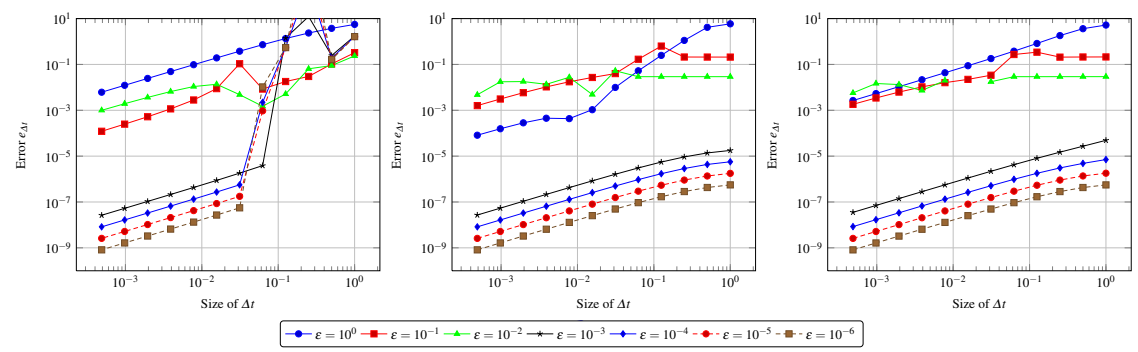

Fig. 3 Numerical results for IMEX-Euler method. Case $v(t=0)=-0.1$ is considered. Left: SPLIT, middle: Implicit, right: RS-IMEX. Values not plotted are NaN, which show an instability of the method.
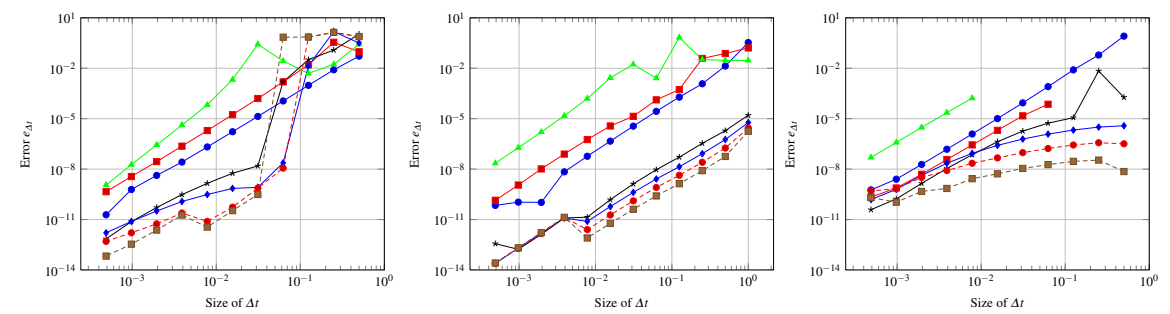

$\rightarrow \varepsilon=10^{0} \rightarrow \varepsilon=10^{-1} \rightarrow \varepsilon=10^{-2} \rightarrow \varepsilon=10^{-3} \rightarrow \varepsilon=10^{-4} \bullet \varepsilon=10^{-5}-\varepsilon=10^{-6}$

Fig. 4 Numerical results for BPR-353 method. Case $v(t=0)=-0.1$ is considered. Left: SPLIT, middle: Implicit, right: RS-IMEX. Values not plotted are NaN, which show an instability of the method. 


\section{Difficult Asymptotics: $\mathbf{v}(\mathbf{t}=\mathbf{0})=\mathbf{0 . 1}$}

This is certainly the most challenging setting for the RS-IMEX splitting, since the reference solution does not coincide with the $\varepsilon \rightarrow 0$ limit. The implications can clearly be seen in Figs. 5 and 6 . Indeed one can see that the RS-IMEX splitting has huge stability problems for small values of $\varepsilon$ and small values of $\Delta t$. This could be due to the initial layer, but most probably, it is due to the fact that the reference solution is not asymptotically close to the original solution; an assumption that stands at the beginning of the RS-IMEX approach. The other splittings are stable, but perform quite poorly.
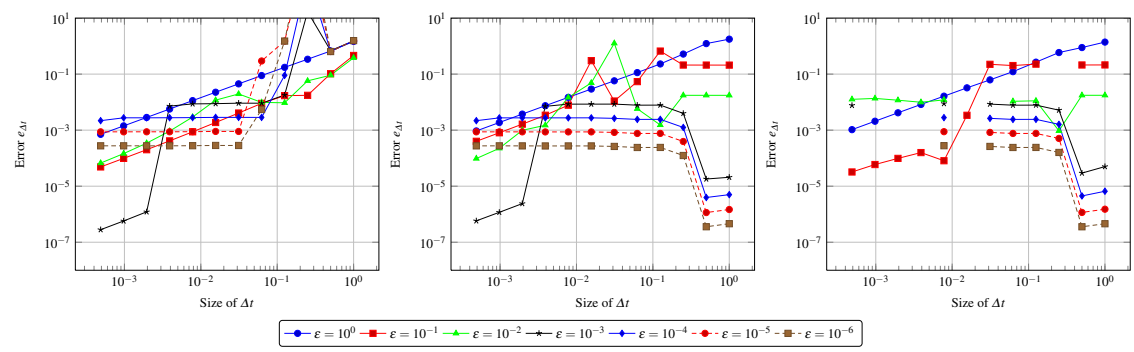

Fig. 5 Numerical results for IMEX-Euler method. Case $v(t=0)=0.1$ is considered. Left: SPLIT, middle: Implicit, right: RS-IMEX. Values not plotted are NaN, which show an instability of the method.
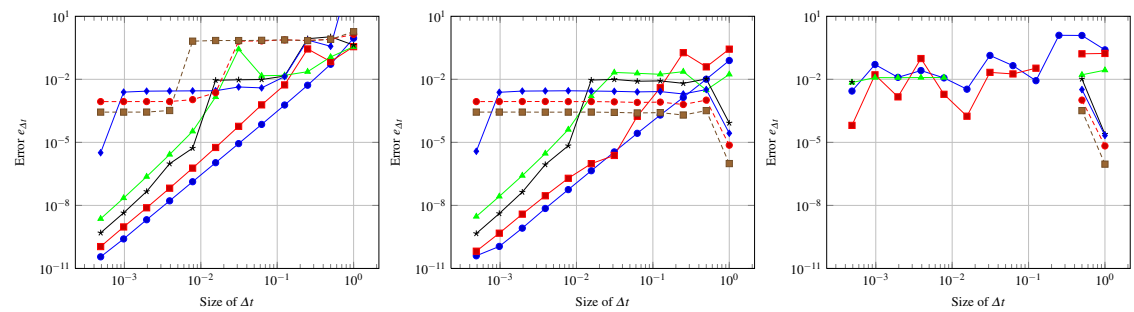

$\rightarrow \varepsilon=10^{0} \rightarrow \varepsilon=10^{-1} \rightarrow \varepsilon=10^{-2} \rightarrow \varepsilon=10^{-3} \rightarrow \varepsilon=10^{-4} \bullet \cdot \varepsilon=10^{-5}-\varepsilon=10^{-6}$

Fig. 6 Numerical results for BPR-353 method. Case $v(t=0)=-0.1$ is considered. Left: SPLIT, middle: Implicit, right: RS-IMEX. Values not plotted are NaN, which show an instability of the method.

The RS-IMEX approach is quite flexible, it allows tuning the reference solution in a certain sense. In particular, one can take the solution that results from taking $v_{(0)}(t=0)=-0.1$, which seems to be a better approximation. Corresponding results are presented in Figs. $\square$ and $\nabla$. The results for the SPLIT splitting and the implicit method (we didn't change anything there) are the same as in the plots before. 
The RS-IMEX splitting, on the other hand, now performs much better. It has some stability issues for large values of $\Delta t$, which can be explained due to the small step size needed to resolve the initial boundary layer. After crossing this critical point the RS-IMEX splitting behaves similar or - in most cases - even better compared to the other splittings.
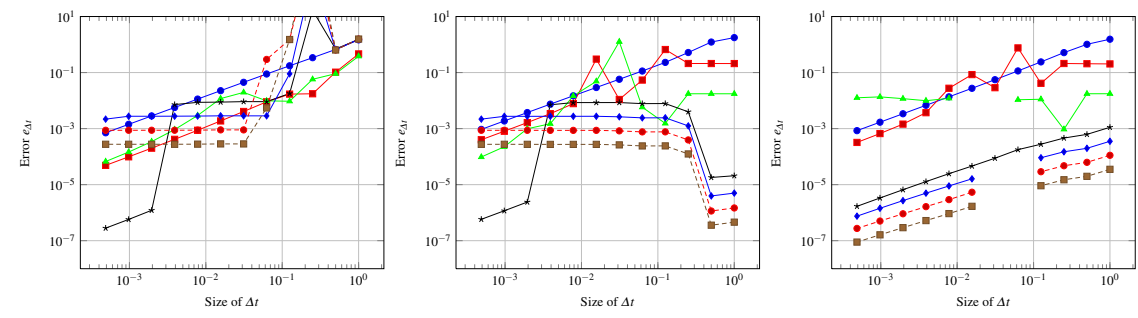

Fig. 7 Numerical results for IMEX-Euler method. Case $v(t=0)=0.1$ is considered. Left: SPLIT, middle: Implicit, right: RS-IMEX. The novelty is here that the RS-IMEX method uses the 'correct' limit solution. Values not plotted are NaN, which show an instability of the method.

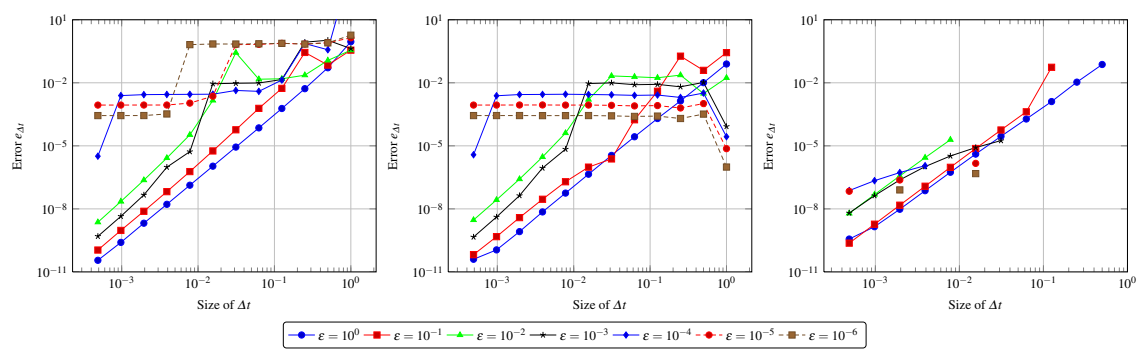

Fig. 8 Numerical results for BPR-353 method. Case $v(t=0)=0.1$ is considered. Left: SPLIT, middle: Implicit, right: RS-IMEX. The novelty is here that the RS-IMEX method uses the 'correct' limit solution. Values not plotted are NaN, which show an instability of the method.

This work is guided by the following questions:

- How does the RS-IMEX scheme behave if unperturbed and perturbed solution are not close to each other?

- In particular, is the RS-IMEX scheme then still uniformly stable as observed in [8]?

- If not, are there potential remedies? 


\section{Conclusion and Outlook}

In this work, we have demonstrated the performance of some stiff ODE solvers, with a focus on the RS-IMEX splitting. In particular, one could observe problems with the RS-IMEX method whenever the asymptotics are not the 'straightforward' way. The flexiblity of the method, however, could in some cases save the day. Of course all this relies on the fact that one is able to identify the correct asymptotics of the problem. This could be achieved by computing the reference solution 'step-by-step' with the possibility of adapting it to the behavior of the approximate solution. Furthermore, it is well-established that such problems should be computed with some sort of adaptivity. Besides doing it via embedded Runge-Kutta methods - which are hard to get for high-order IMEX schemes - one can also use the difference of the solution and the reference solution as an error indicator. Based on this indicator, one can then directly reduce the time step or switch to another, more suited, method. This is in particular important as a step toward all-Mach schemes for the NavierStokes equations, where the (local) speed of the flow (i.e., in our setting the value of $\varepsilon$ ) can be small in one and quite large in another region.

Acknowledgements We thank Peter de Maesschalck for fruitful discussions. The first author has been partially supported by the German Research Foundation (DFG) project NO 361/3-3, and the University of Hasselt in the framework of the BOF 2016.

\section{References}

1. Ascher, U. M., Ruuth, S. and Spiteri, R: Implicit-explicit Runge-Kutta methods for timedependent partial differential equations. Appl. Numer. Math. 25, 151-167 (1997).

2. Ascher, U. M., Ruuth, S and Wetton, B: Implicit-Explicit methods for time-dependent partial differential equations. SIAM J. Numer. Anal. 32, 797-823 (1995) .

3. Boscarino, S: Error analysis of IMEX Runge-Kutta methods derived from differentialalgebraic systems. SIAM J. Numer. Anal. 45, 1600-1621 (2007).

4. Boscarino, S, Pareschi, L. and Russo, G.: Implicit-explicit Runge-Kutta schemes for hyperbolic systems and kinetic equations in the diffusion limit. SIAM J. Sci. Comput. 35 (1), A22A51 (2013)

5. De Maesschalck, P., Kutafina, E. and Popović, N.: Sector-delayed-Hopf-type mixed-mode oscillations in a prototypical three-time-scale model. Appl. Math. Comput. 273, 337-352 (2016).

6. Kaiser, K. and Schütz, J.: A high-order method for weakly compressible flows. IGPM Preprint Nr. 456 (2016) (submitted for publication)

7. Kaiser, K., Schütz, J., Schöbel, R. and Noelle, S.: A new stable splitting for the isentropic Euler equations. J. Sci. Comput. (2016). doi:10.1007/s10915-016-0286-6 (in press)

8. Schütz, J. and Kaiser, K.: A new stable splitting for singularly perturbed ODEs. Appl. Numer. Math. 107, 18-33 (2016). 